\title{
Detection probability evaluation method for infrared point target under complicated backgrounds
}

\author{
by Haichao Zheng*, Xia Mao*, Lin Jiang* and Yuli Xue*
}

*School of Electronic and Information Engineering, Beihang University, Beijing 10019, P. R. China, moukyoucn@yahoo.com.cn

\begin{abstract}
Traditional detection probability evaluation method for infrared point target is not appropriate in the condition of complicated background due to the influence of background radiance. Firstly, this paper deduces the traditional detection probability evaluation method for point target under unitary background and analyzes its defects. Then, this paper introduces the idea of probability distribution into the traditional method and proposes a novel detection probability evaluation method under complicated backgrounds. Finally, a detection probability calculation instance shows that the new method can accurately estimate the detection probability under complicated backgrounds.
\end{abstract}

\section{Introduction}

Infrared (IR) imaging technique is widely used in military fields, such as airborne fire control, ground-based antiaircraft systems and guided missiles. For an IR imaging system, detection probability is a crucial technique performance parameter. Scholars have carried out some researches and proposed human-in-the-loop detection probability evaluation models [1,2]. However, there is not an in-depth research on detection probability of IR automatic target recognition system. Traditional detection probability evaluation method has been derived to estimate detection probability of IR automatic target recognition system [3]. Nevertheless, traditional evaluation method only considered the design principles of system, while ignoring the external factors, especially the IR radiances of complicated backgrounds. Traditional evaluation model is valid only when the background is unitary, otherwise the calculated detection probability value will deviate from the true value due to the influence of background radiance.

To solve this problem, we firstly analyze the effects of complicated backgrounds on detection probability, and then introduce the idea of probability distribution to improve the traditional detection probability equation. In our model background radiance and detection probability are respectively changed from a single value to a vector with dimensions calculated according to the gray distribution of background image. After that, the background radiance can be obtained through background gray information. Finally, a novel model valid for various backgrounds is deduced.

\section{Traditional detection probability model and limitation analysis}

With the development of manufacture technology, the main noise of an IR imaging system is the noise of detector, which is in concordance with Gaussian distribution. When a specific external signal is collected together with noise, the output signal of detector still meets Gaussian distribution. Its probability density is:

$$
p_{d}(V)=\frac{1}{\sqrt{2 \pi} V_{n}} \exp \left(-\frac{\left(V-V_{a}-V_{s}\right)^{2}}{2 V_{n}^{2}}\right)
$$

Where $V_{s}$ is the external input signal voltage, $V_{a}$ is the mean of signal voltage, $V_{n}$ is the root mean square of the detector's noise voltage.

Detection probability is mainly related to Signal-to-Noise Ratio (SNR) and Threshold Signal-to-Noise Ratio (TNR). For a detector, detection probability is the probability that SNR exceeds TNR. Therefore, we can get the detection probability equation through the integration of Eq. (1).

$$
P_{d}\left(S_{N R}\right)=\frac{1}{\sqrt{2 \pi}} \int_{-\infty}^{S_{N R}-T_{N R}} \exp \left(-\frac{\rho^{2}}{2}\right) d \rho
$$

Where $S_{N R}=V_{s} / V_{n}$ is the input SNR of detector, $T_{N R}=\left(V-V_{a}\right) / V_{n}$ is the TNR of detector, $\rho$ is the integration variable, which is equal to $\left(V-V_{a}-V_{s}\right) / V_{n}$.

In general, the TNR is defined as the specific SNR when the detection probability is $50 \%$. When TNR is fixed, the relationship between detection probability and SNR is shown in figure 1. 


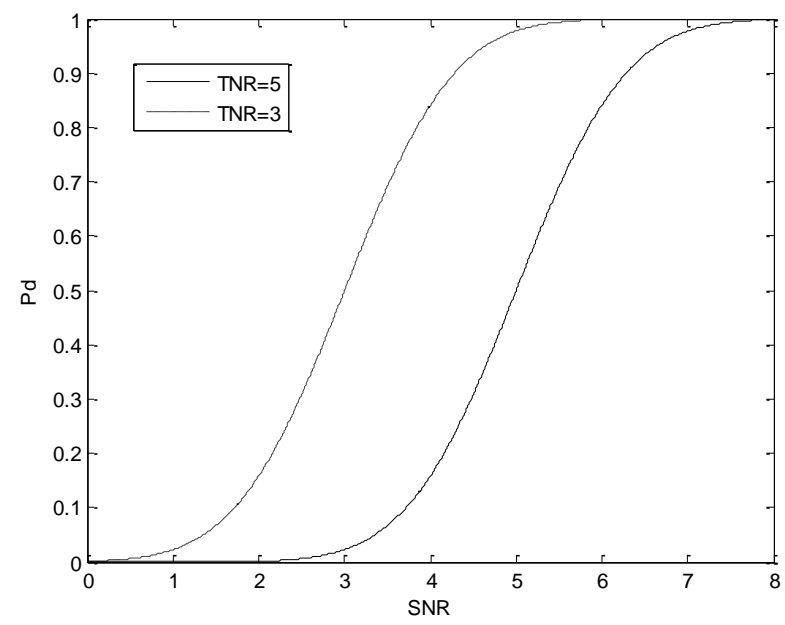

Fig.1. Illustration of relationship between detection probability and SNR

Figure 1 shows that the detection probability of an IR imaging system is a monotonically increasing function of SNR. When the SNR is fixed, the detection probability increases as the TNR value decreases.

For an IR imaging system, SNR is related to the radiation of target, background radiance, and the distance between target and IR imaging system. Operating distance equation for an IR point target is given under detector noise limitation as shown in Eq. (3) $[4,5,6]$.

$$
R=\left[\frac{A_{o}\left(N_{t}-N_{b}\right) A \tau_{o} \tau_{a} D^{*} \delta}{\left(V_{s} / V_{n}\right)\left(A_{d} \Delta f\right)^{1 / 2}}\right]^{\frac{1}{2}}
$$

Where $R$ is the operating distance of IR imaging system, $A_{o}$ is the effective area of optical system, $N_{t}$ is the average spectral radiance produced by target, $N_{b}$ is the average spectral radiance produced by background, $A$ is the effective area of target, $\tau_{o}$ is the average spectral transmittance of optical system, $\tau_{a}$ is the average spectral transmittance of atmosphere, $D^{*}$ is the average normalized spectral detectability, $\delta$ is the signal attenuation factor, $V_{s} / V_{n}$ is SNR, $A_{d}$ is the effective area of detector, $\Delta f$ is the equivalent bandwidth of noise of IR imaging system. Therefore, the SNR equation can be derived from Eq. (3).

$$
S_{N R}\left(N_{t}, N_{b}, R\right)=\frac{A_{o}\left(N_{t}-N_{b}\right) A \tau_{o} \tau_{a} D^{*} \delta}{\left(A_{d} \Delta f\right)^{1 / 2} \cdot R^{2}}
$$

In Eq. (4), once $R$ and $N_{b}$ have been identified, SNR is only related to background radiance. So Eq. (4) can be changed to:

$$
S_{N R}\left(N_{b}\right)=\frac{A_{o}\left(N_{t}-N_{b}\right) A \tau_{o} \tau_{a} D^{*} \delta}{\left(A_{d} \Delta f\right)^{1 / 2} \cdot R^{2}}
$$

Substituting Eq. (5) into Eq. (2), we can conclude the detection probability equation as Eq. (6) after rearranging

$$
P_{d}\left[S_{N R}\left(N_{b}\right)\right]=\frac{1}{\sqrt{2 \pi}} \int_{-\infty}^{S_{N R}\left(N_{b}\right)-T_{N R}} \exp \left(-\frac{\rho^{2}}{2}\right) d \rho
$$

Eq. (6) indicates that traditional method ignores the diversification of background, and only calculates a specific value to represent detection probability, which is apparently unreliable under a complicated background. Image with a complicated background often has plentiful gray values and disordered spatial distribution of pixels. Obviously, the IR radiant ability of a complicated background is not fixed considering the one-to-one relationship between imaging gray value and IR radiant ability. Therefore, under a complicated background, a target may be shielded by the background fluctuation, while it is still possible to be detected when the background region's radiance is small enough. To exactly solve the negative effect of complicated backgrounds, it is more reasonable that the detection probability should be represented by multi-values. 


\section{New detection probability model}

Figure 2 shows four complicated background IR images and their gray value distribution: (a) sea clutter, (b) skysea, (c) cloudy sky, (d) complicated terrain.
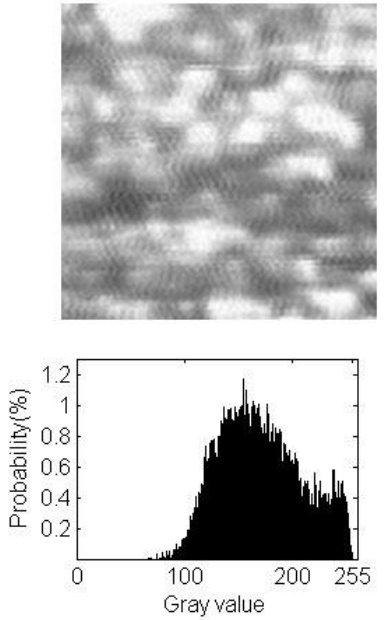

(a)
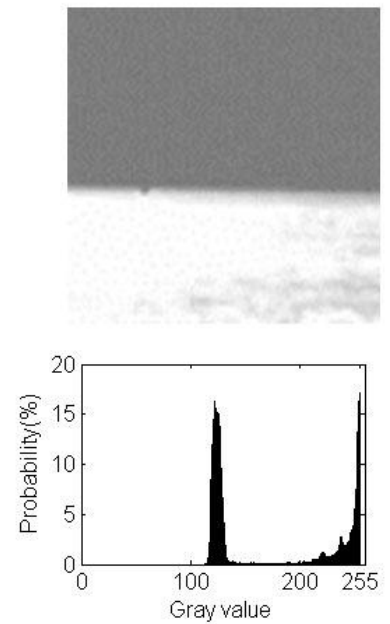

(b)
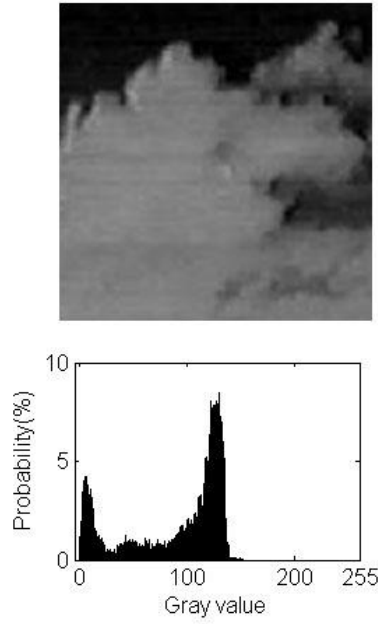

(c)
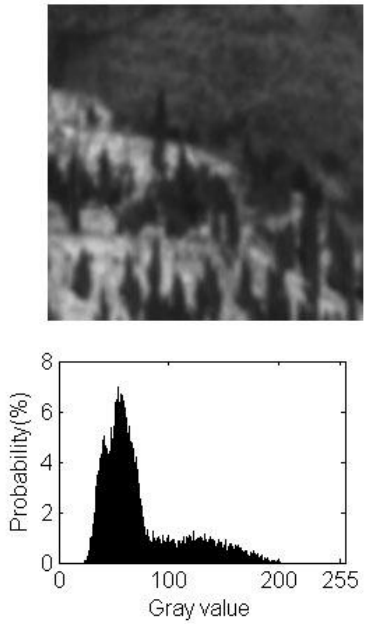

(d)

Fig.2. Four complicated background IR images and their gray value distribution

From the images in figure 2, it can be seen that although each image is complex, the number of its gray values is fixed for a certain imaging system with constant imaging gray levels. Furthermore, the proportion of each gray value is certain, which indicates that background radiance has several possible values with fixed number and certain distribution according to the one-to-one relationship between imaging gray value and IR radiance. Considering the influence of background IR radiance on detection probability, it can be concluded that: detection probability of the IR imaging system on a target has several possible values with fixed numbers and the probability of each possible value is certain.

Therefore, this paper introduces the idea of probability distribution into the traditional equation. In this method, background radiance, SNR and detection probability are changed from a single value to a vector respectively and endued with a property named occurrence probability. The dimension of SNR vector and detection probability vector is equal to that of background radiance vector. For instance, if there are 10 possible background IR radiance values, the dimension is 10. Then the SNR equation can be changed to Eq. (7).

$$
S_{N R}\left(P_{i}\right)=\frac{A_{o}\left(N_{t}-N_{b}\left(P_{i}\right)\right) A \tau_{o} \tau_{a} D^{*} \delta}{\left(A_{d} \Delta f\right)^{1 / 2} \cdot R^{2}} \quad i=1,2, \ldots, m
$$

Where $\left[S_{N R}\left(P_{i}\right)\right]_{i=1,2, \ldots, m}$ is the SNR vector, $\left[N_{b}\left(P_{i}\right)\right]_{i=1,2, \ldots, m}$ is the background radiance vector, $P_{i}$ is the occurrence probability of the $i^{\text {th }}$ element of the vector, which satisfies $\sum_{i=1}^{M} P_{i}=1$.

Then, the integrated detection probability equation is:

$$
P_{d}\left(P_{i}\right)=\frac{1}{\sqrt{2 \pi}} \int_{-\infty}^{S_{N R}\left(P_{i}\right)-T_{N R}} \exp \left(-\frac{\rho^{2}}{2}\right) d \rho \quad i=1,2, \ldots, m
$$

For the new equation Eq. (8), when the background is unitary, the dimension of detection probability vector is 1 , and the new equation is degraded into Eq. (2). So the traditional operating distance equation is a special case of the new equation.

\section{Method for calculating the IR radiance of complicated background}

In our new equation, the key is to obtain the information of background radiance, including the number of possible background radiance values, each possible value as well as its corresponding occurrence probability. In practical application, it is hard to obtain the substance component and lighting conditions of a complicated background. Thus IR radiance of a complicated background is difficult to be calculated correctly by traditional methods, which focus on establishing background IR radiance model $[7,8]$. In order to solve this problem, we use an approach proposed in our previous paper to calculate the background IR radiance via its imaging gray information [9]. 
After overcoming the atmosphere attenuation, IR radiance of the target reaches the IR imaging system, and then is transformed to grayscale image. The work flow for calculating IR radiance of a complicated background in this paper is the reverse imaging process of the imaging system.

The equation of calculating background IR radiance is:

$$
N_{b}\left(P_{i}\right)=N_{b_{-} o p t}\left(P_{i}\right) / \tau_{a}
$$

Where $\left[N_{b}\left(P_{i}\right)\right]_{i=1, \ldots, m}$ is the background IR radiance responding to imaging gray value, $\left[N_{b_{-} o p t}\left(P_{i}\right)\right]_{i=1, \ldots, m}$ is the background IR radiance at the entrance pupil of the imaging system. $\tau_{a}$ is atmospheric transmittance calculated by the atmosphere transmission calculation software LOWTRAN7. The inputs of software are the environment parameters recorded when IR image is took by the IR imaging system.

Although the relationship between IR radiance value and imaging gray value is non-linear, it is ascertained and can be described when IR imaging system's aperture is fixed on. Here the relationship between IR radiance value and imaging gray value can be expressed by Radiance-Grey function. In this paper, "black-box" is introduced to describe the relationship, which is confirmed by the inputs and outputs while neglecting the detailed process. Here, we describe Radiance-Gray function by Taylor series as following:

$$
N=A_{0}+A_{1} \cdot G+A_{2} \cdot G^{2}+A_{3} \cdot G^{3}+\ldots+A_{n} \cdot G^{n}
$$

Where $N$ is the radiance value, $G$ is the corresponding gray value, $A_{0}, A_{1} \ldots A_{n}$ is the corresponding Taylor coefficients. The Taylor coefficients of the function are determined by the following steps: firstly, shoot close-up of a blackbody simulator in 20 different temperatures using the IR imaging system and record 20 sets of samples comprising the temperature value of blackbody simulator, IR radiance value and imaging gray value; secondly, fill IR radiance value and gray value of each sample into Radiance-Gray function; finally, calculate the coefficients using Least-Squares Estimation.

Through experimental validation, the coefficients nearly equal to 0 after the fifth order, so we use fifth-order Taylor series to describe the Radiance-Gray function. In our experiments with 30 sets of samples, the mean difference between IR radiance calculated by the function and the actual value is 3.4\%. The experimental result shows that, for an imaging system with fixed aperture and grey-levels, it is appropriate for us to describe the relationship between imaging gray value and corresponding IR radiance at the entrance of the system.

\section{Experimental result and discussion}

In order to validate our new detection probability evaluation method, this paper calculates the detection probability of a medium wave IR imaging system upon cruise missile under a complicated terrain background as shown in figure 2(d), while the flight velocity of the missile is $0.75 \mathrm{Ma}$ (speed of sound) and the grey-levels of the image taken by our imaging system are in the range 0 255. The parameters of the IR imaging system are specified as: TNR: 5, operating distance: $9400 \mathrm{~m}$. And the detection probability is $50 \%$ when the background is unitary.

First of all, the Radiance-Gray function is ascertained by the approach mentioned above. Here, we used 20 sets of samples, and the received function is shown in figure 3.

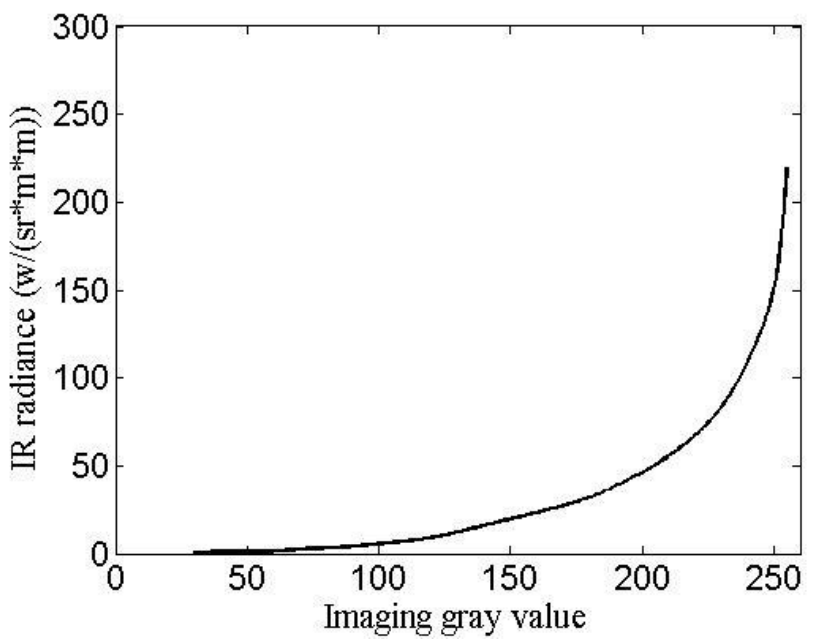

Fig.3. Illustration of Radiance-Grey function

Then, IR image under a complicated background is taken and its gray vector and background IR radiance vector are obtained using our method. The IR radiance vector for terrain background is shown in figure 4 . 


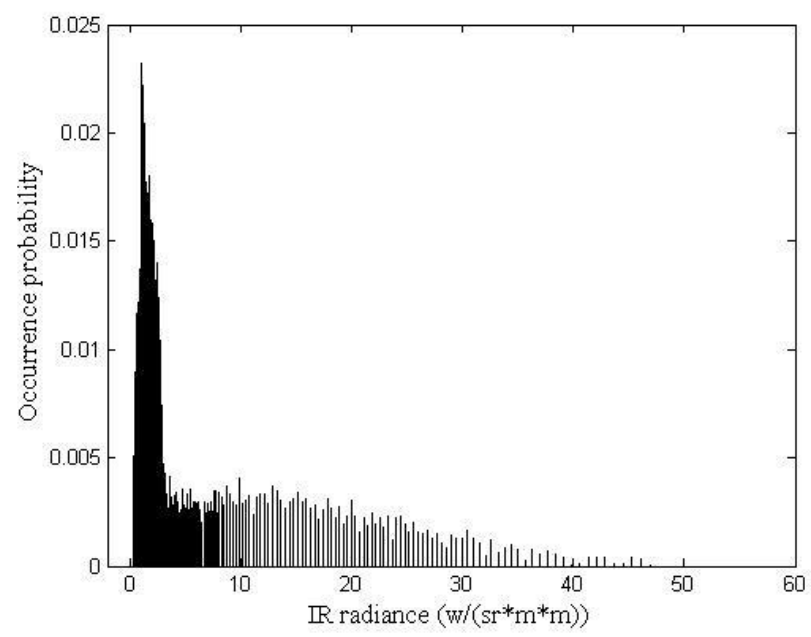

Fig.4. IR radiance of complicated terrain background

After that, IR radiance vector and target radiance are filled into the new detection probability equation. Finally, the detection probability vector is calculated as shown in figure 5 , wherein $x$-axis represents the detection probability value and $y$-axis is the corresponding occurrence probability.

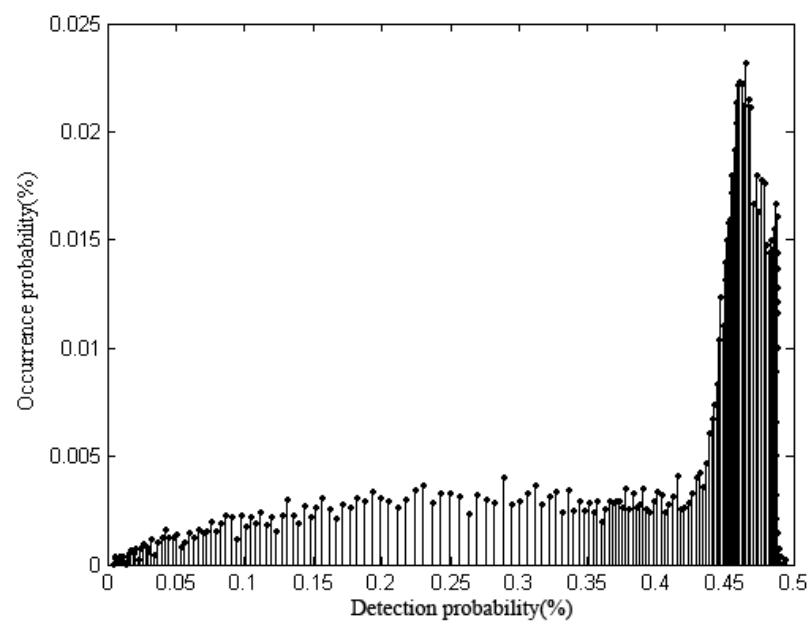

Fig.5. Detection probability

The experimental result shows that, under the complicated terrain background, the detection probability of the IR imaging system for point target has 179 possible values, which are all less than the theoretical value (50\%), and each value has its own probability. In figure 2(d), we note that the components of the histogram are concentrated on the low side of the gray scale, which indicates that the IR radiance of complicated terrain background is not very strong. Consequently, the probability that detection probability values distributed between $45 \%$ and $50 \%$, accounts for $80 \%$, which is close to the actual situation. This result is consistent with our prediction that detection probability is multi-values and all less than the theoretical value when background is complicated. The calculation instance proves that the new method proposed in this paper could solve the detection probability evaluation problem for an IR imaging system under complicated backgrounds.

\section{Conclusions}

In this paper, we deduce the detection probability equation for IR point target under a unitary background from operating distance equation. After analyzing the effects of complicated backgrounds on detection probability, we propose a new detection probability evaluation method under complicated backgrounds by introducing the idea of probability distribution. Experimental result shows that our new detection equation realizes the detection probability evaluation under both unitary background and complicated background. 


\section{REFERENCES}

[1] Thomas J.Meitzler, Grant G.Gerhart, E.Sohn, H.Singh, "Detection probability using relative clutter in infrared images". IEEE Transactions on Aerospace and Electronic Systems, vol. 34, pp. 955-962, 1998.

[2] Grant R.Gerhart, Edwina L.Bednarz, Thomas J.Meitzler, Euijung Sohn, Robert E.Karlsen, "Target acquisition methodology for visual and infrared imaging sensors". Optical Engineering, vol. 35, pp. 3026-3036, 1996.

[3] Runshun Li, Xiangyan Yuan, Zhigang Fan, Baojun Zuo, "Operating range prediction of infrared imaging system". Infrared and Laser Engineering, vol. 30, pp. 1-3, 2001.

[4] Hundson, R.D., "Infrared System Engineering". pp. 62-91, John Wiley and Sons, New York, 1969.

[5] Hanping Wu, Xinjian Yi, "Operating distance equation and its equivalent test for infrared search system with full orientation”. International Journal of Infrared and Millimeter Waves, vol. 24, pp. 2059-2068, 2003.

[6] Datta P.K., Kumar A., Nijhawan O. P., "Range estimation of thermal imaging system from MRTD and MTF measurement". Optik, vol. 97, pp. 1-3, 1994.

[7] Mei Wang, Guotu Shen, Baocheng Yang, Jiguang Cai, Jing Gao, Zhanhai Dong, "Simulation of infrared images of small target and sea and sky background”. Acta Armamentarii, vol. 28, pp. 561-564, 2007.

[8] Yimin Xuan, Decang Li, Yuge Han, "Synthetic infrared image of complicated terrain background". Journal of Infrared and Millimeter Waves, vol. 21, pp. 133-136, 2002.

[9] Weihe Diao, Xia Mao, Le Chang, Lin Jiang, "Operating distance evaluation method for infrared imaging system under complicated backgrounds". Electronics Letters, vol. 45, pp. 1309-1310, 2009. 